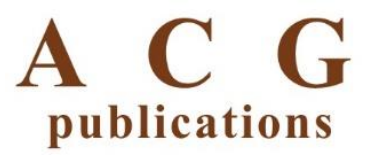

Org. Commun. 14:2 (2021) 133-143

organic

communications

\title{
Discovery of new capsaicin and dihydrocapsaicin derivatives as histone deacetylase inhibitors and molecular docking studies
}

\author{
Pakit Kumboonma ${ }^{1 *}$, Somprasong Saenglee ${ }^{2}$, Thanaset Senawong \\ and Chanokbhorn Phaosiri ${ }^{4}$
}

\author{
${ }^{1}$ Department of Applied Chemistry, Faculty of Science and Liberal Arts, \\ Rajamangala University of Technology Isan, Nakhon Ratchasima, 30000 Thailand \\ ${ }^{2}$ Ban Dong Subdistrict Administration Organization, Ubolratana District, Khon Kaen, 40250 Thailand \\ ${ }^{3}$ Natural Products Research Unit, Department of Biochemistry, Faculty of Science, \\ Khon Kaen University, Khon Kaen, 40002 Thailand \\ ${ }^{4}$ Natural Products Research Unit, Center of Excellence for Innovation in Chemistry, Department of \\ Chemistry, Faculty of Science, Khon Kaen University, Khon Kaen, 40002 Thailand
}

(Received March 03, 2021; Revised May 03, 2021 ; Accepted May 12, 2021)

\begin{abstract}
Capsaicin and dihydrocapsaicin were modified at phenolic moieties and allylic of double-bond to provide seven capsaicin and dihydrocapsaicin derivatives. Their structures were assigned by spectroscopic techniques (1D-NMR and MS). All compounds were evaluated as histone deacetylase inhibitors via in vitro fluorometric assay at $100 \mu \mathrm{M}$. The results revealed that the chloranil derivatives were found to be best histone deacetylase inhibitors among the tested compounds with $86 \%$ and $87 \%$ inhibitions. In addition, molecular docking experiments of the active compounds with representatives of class I (HDAC1, HDAC2, HDAC3 and HDAC8), class IIa (HDAC4 and HDAC7) and class IIb (HDAC6) HDAC isoforms displayed potential isoform-selective HDAC inhibitors. Those data show a new method for providing the isoform-selective histone deacetylase inhibitors from common natural products.
\end{abstract}

Keywords: Red chili pepper; Capsicum annuum; histone deacetylase; HeLa cell; cytotoxiciy; molecular docking; (C) 2021 ACG Publications. All rights reserved.

\section{Introduction}

Modification of histone proteins plays a critical role in the epigenetic regulation of transcription in cell. Histone acetylation is controlled by a balance between the opposing functions of histone acetyl transferases (HATs) and Histone deacetylases (HDACs). The balance between the activities of HATs

\footnotetext{
* Corresponding author: E-mail: pakit.ku@rmuti.ac.th

The article was published by ACG Publications 
New capsaicin and dihydrocapsaicin derivatives as histone deacetylase inhibitors

and HDACs are associated with cancer development involved in the control of the cell cycle progression, differentiation, apoptosis and tumourigenesis. Therefore, HDACs have become an essential therapeutic target for a broad range of human disorders such as cancers, diabetes and brain disorders ${ }^{1-4}$. Based on sequence homology to yeast models, eighteen mammalian isoforms HDACs are grouped into four classes $^{5}$. Class I (HDAC1, 2, 3 and 8), class IIa (HDAC4, 5, 7 and 9), class IIb (HDAC6 and 10) and class IV (HDAC11), HDACs are zinc dependent metallo proteins. Class III (SIRT1-7), HDACs are $\mathrm{NAD}^{+}$-dependent proteins. The HDAC isoforms are involved in multiple different stages and types of diseases and cancers. For example, high expression of HDAC1, HDAC2 and HDAC 3 are associated with poor outcomes in ovarian, pancreatic and lung cancers ${ }^{6,7}$. High expression of HDAC8 correlates with advanced stage disease and poor survival in neuroblastoma ${ }^{8,9}$. HDAC2 have also been detected in neurodegenerative diseases, ovarian and gastric cancers ${ }^{10,11}$. HDAC1 and HDAC5 have been detected in gastric cancer whereas HDAC2 and HDAC 3 have also been found in colorectal cancer ${ }^{12,13}$. HDAC4 have been associated with colon cancer, ovarian cancer, and gastric cancer ${ }^{14-16}$. HDAC7 plays a major role in cardiovascular developments and diseases ${ }^{17}$.

Histone deacetylase inhibitors contribute to tumor cell growth arrest, differentiation, enhancing cell apoptosis and promoting cell-cycle arrest by blocking substrate access to the histone deacetylaseactive site ${ }^{3}$. The structure-activity relationships and X-ray crystallography of HDAC inhibitors reveal that HDACs inhibitors are composed of a metal-binding domain, a linker domain, and a surfacerecognition domain. The metal-binding functional group is responsible for binding a $\mathrm{Zn}^{2+}$ ion in the HDAC active site, whereas the hydrocarbon linker domain plays a role in filling out a narrow tunnel leading to the active site. Finally, the capping group on the surface-recognition domain interacts with the amino acids close to the entrance of the HDAC active site. Capsaicin (CAP) and dihydrocapsaicin (DHC) partially mimic the structure of the FDA-approved HDAC inhibitors suberoylanilide hydroxamic acid (SAHA) and trichostatin A (TSA). Therefore, capsaicin and dihydrocapsaicin derivatives may also inhibit histone deacetylases.

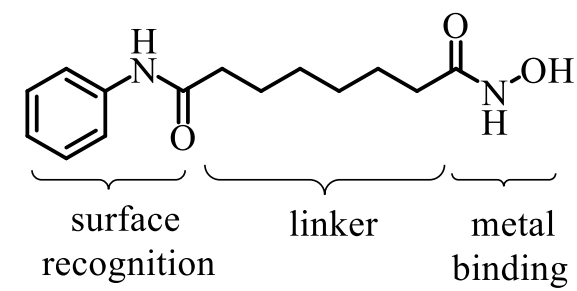

SAHA, $\mathrm{IC}_{50}=110-370 \mathrm{nM}$<smiles>COc1cc(CNC(=O)CCCC/C=C/C(C)C)ccc1O</smiles>

Capsaicin (1)<smiles>CC(/C=C/C(=O)NO)=C\C(C)C(=O)c1ccc(N(C)C)cc1</smiles>

$\mathrm{TSA}, \mathrm{IC}_{50}=12 \mathrm{nM}$<smiles>COc1cc(CNC(=O)CCCCCCC(C)C)ccc1O</smiles>

Dihydrocapsaicin (2)

Figure 1. The pharmacophoric summary of HDAC inhibitor structures in comparison to capsaicin and dihydrocapsaicin

In this work, the structural modifications of CAP and DHC at the metal-binding domain and a surface-recognition domain were prepared to improve HDAC inhibitory activity. In order to search for the isoform-selective inhibitors with different HDAC enzymes, molecular docking studies of all derivatives with HDAC1, 2, 3, 4, 6, 7 and HDAC 8 isoforms are described. These derivatives exhibit promising HDAC inhibition with high selective inhibitors and may warrant further studies at the cellular level.

\section{Experimental}




\subsection{Chemical Material and Apparatus}

Reagents and solvents were purchased from Sigma-Aldrich Corporation (USA). All solvents and volatile reagents were distilled prior to use. Reactions were monitored using analytical TLC plates (silica gel $60 \mathrm{~F}_{254}$, Merck (Germany)). Silica gel grade 60 (230-400 mesh, Merck (Germany) was used for column chromatography. NMR spectra were recorded in the indicated solvents on a Varian Mercury plus spectrometer operating at $400 \mathrm{MHz}\left({ }^{1} \mathrm{H}\right)$ and at $100 \mathrm{MHz}\left({ }^{13} \mathrm{C}\right)$. IR spectra were obtained on Perkin Elmer Spectrum One FT-IR spectrophotometer. Mass spectra were determined by using a Micromass Q-TOF 2 hybrid quadrupole, time-of-flight (Q-TOF) mass spectrometer with a Z-spray ES source.

\subsection{Separation of Capsaicin and Dihydrocapsaicin}

One kilogram of dried red chili spur peppers (Capsicum annuum) was extracted with $\mathrm{CH}_{2} \mathrm{Cl}_{2}$ and purified by column chromatography as previously described to provide a mixture of Capsaicin $(\mathrm{CAP}, 1)$ and Dihydrocapsaicin (DHC, 2) $(690 \mathrm{mg})$ as a yellow-orange oil ${ }^{18}$.

\subsection{Chemistry}

\subsubsection{Preparation of Silyl Ether-CAP (3) and Silyl Ether-DHC (4)}

To a suspension of $\mathrm{NaH}(60 \%$ in mineral oil, $6.20 \mathrm{mg}, 0.16 \mathrm{mmol}, 2$ equis.) in THF (1 mL) at 0 ${ }^{\circ} \mathrm{C}$ under $\mathrm{N}_{2}$ was added the solution of 1 and 2 mixture (1:1), (23.70 mg, $\left.0.08 \mathrm{mmol}\right)$ in THF $(2 \mathrm{~mL})$ over a $15 \mathrm{~min}$ period. The reaction mixture was warmed to room temperature and stirred for $10 \mathrm{~min}$ before TBDMSCl (15.1 mg, $0.1 \mathrm{mmol}, 1.3$ equis) in THF $(1 \mathrm{~mL})$ was added over $5 \mathrm{~min}$. The mixture was refluxed for $29 \mathrm{~h}$. After the solution was cooled to ambient temperature, 5\% $\mathrm{NaHCO}_{3}(4 \mathrm{~mL})$ was added slowly to the reaction mixture followed by extraction with EtOAc $(3 \times 20 \mathrm{~mL})$. The combined organic layers were dried and concentrated. Purification of the crude product by column chromatography (1:3 (v/v) EtOAc/hexanes) gave an orange oil of compounds 3 and 4 (24.5 mg, 96\%).

(E)-N-(4-(tert-butyldimethylsilyloxy)-3-methoxybenzyl)-8-methylnon-6-enamide (3): $R_{f}=0.85(1: 1(\mathrm{v} / \mathrm{v})$ EtOAc/hexanes). IR (neat) : 3290, 2923, 1645, 1515, 1286, 1159, 1126, 1036, 902, $840 \mathrm{~cm}^{-1}$. ${ }^{1} \mathrm{H}$ NMR $\left(\mathrm{CDCl}_{3}\right): \delta 6.79\left(\mathrm{~d}, J=8.4 \mathrm{~Hz}, 1 \mathrm{H}_{5}\right), 6.75\left(\mathrm{~s}, 1 \mathrm{H}_{2}\right), 6.70\left(\mathrm{~d}, J=8.4 \mathrm{~Hz}, 1 \mathrm{H}_{6}\right), 5.70\left(\mathrm{br} \mathrm{s}, 1 \mathrm{H}_{8}\right), 5.35(\mathrm{~m}$, $\left.1 \mathrm{H}_{14}\right), 5.30\left(\mathrm{~m}, 1 \mathrm{H}_{15}\right), 4.38\left(\mathrm{~d}, J=5.4 \mathrm{~Hz}, 2 \mathrm{H}_{7}\right), 3.79\left(\mathrm{~s}, \mathrm{OCH}_{3}\right), 2.20\left(\mathrm{t}, J=7.8 \mathrm{~Hz}, 2 \mathrm{H}_{10}\right), 1.98(\mathrm{q}, J=$ $7.2 \mathrm{~Hz}, 2 \mathrm{H}_{13}$ ), 1.64 (quin, $\left.J=7.8 \mathrm{~Hz}, 2 \mathrm{H}_{11}\right), 1.40$ (quin, $\left.J=7.8 \mathrm{~Hz}, 2 \mathrm{H}_{12}\right), 1.29\left(\mathrm{~m}, 1 \mathrm{H}_{16}\right), 1.00\left(\mathrm{~s}, 9 \mathrm{H}_{21}\right)$, $0.95\left(\mathrm{~d}, J=6.6 \mathrm{~Hz}, 6 \mathrm{H}_{17,18}\right), 0.18\left(\mathrm{~s}, 6 \mathrm{H}_{19}\right) .{ }^{13} \mathrm{C} \mathrm{NMR}\left(\mathrm{CDCl}_{3}\right): \delta 172.52\left(\mathrm{C}_{9}\right), 150.62\left(\mathrm{C}_{3}\right), 144.03\left(\mathrm{C}_{4}\right)$, $137.61\left(\mathrm{C}_{15}\right), 131.31\left(\mathrm{C}_{1}\right), 126.01\left(\mathrm{C}_{14}\right), 120.38\left(\mathrm{C}_{6}\right), 119.68\left(\mathrm{C}_{5}\right), 111.48\left(\mathrm{C}_{2}\right), 55.01\left(\mathrm{OCH}_{3}\right), 43.03$ $\left(\mathrm{C}_{7}\right), 36.21\left(\mathrm{C}_{10}\right), 31.75\left(\mathrm{C}_{16}\right), 29.16\left(\mathrm{C}_{13}\right), 28.81\left(\mathrm{C}_{12}\right), 25.37\left(\mathrm{C}_{21}\right), 24.84\left(\mathrm{C}_{11}\right), 22.18\left(\mathrm{C}_{17}\right), 22.18\left(\mathrm{C}_{18}\right)$, $17.97\left(\mathrm{C}_{20}\right),-5.11\left(\mathrm{C}_{19}\right)$.

\subsubsection{Preparation of Allylic Alcohol Silyl Ether (5)}

To a solution of silyl ether-CAP (3) and silyl ether-DHC (4) $(60 \mathrm{mg}, 0.14 \mathrm{mmol})$ in THF ( $3 \mathrm{~mL})$ was added $\mathrm{SeO}_{2}(23 \mathrm{mg}, 1.5$ equis). Catalytic amount of pyridine $(0.1 \mathrm{~mL})$ was added and the reaction mixture was heated to reflux for $10 \mathrm{~h}$. Then water $(3 \mathrm{~mL})$ was added and the mixture was extracted with EtOAc $(3 \times 20 \mathrm{~mL})$. The organic layer was combined, washed with water, brine and dried with anhydrous $\mathrm{Na}_{2} \mathrm{SO}_{4}$. The solvent was removed to furnish crude mixture. Purification of the crude mixture by column chromatography (1:1 (v/v) EtOAc/hexanes) gave a yellow oil of compound $5(25 \mathrm{mg}, 80 \%)$ and recovered compound $\mathbf{4}(28 \mathrm{mg}, 93 \%)$.

(E)-N-(4-(tert-butyldimethylsilyloxy)-3-methoxybenzyl)-5-hydroxy-8-methylnon-6-enamide (5): $\mathrm{R}_{f}=$ $0.40(1: 1(\mathrm{v} / \mathrm{v})$ EtOAc/hexanes). IR (neat) : 3306, 2928, 2856, 1645, 1515, 1462, 1283, 1158, 1126, 1037, 902, 840, $782 \mathrm{~cm}^{-1} .{ }^{1} \mathrm{H}$ NMR $\left(\mathrm{CDCl}_{3}\right): \delta 6.78\left(\mathrm{~d}, J=8.0 \mathrm{~Hz}, 1 \mathrm{H}_{5}\right), 6.76\left(\mathrm{~s}, 1 \mathrm{H}_{2}\right), 6.70(\mathrm{~d}, J=8.0$ $\left.\mathrm{Hz}, 1 \mathrm{H}_{6}\right), 5.80\left(\right.$ br s, $\left.1 \mathrm{H}_{8}\right), 5.60\left(\mathrm{~m}, 1 \mathrm{H}_{15}\right), 5.40\left(\mathrm{dd}, J=7.0,15.0 \mathrm{~Hz}, 1 \mathrm{H}_{14}\right), 4.35\left(\mathrm{~d}, J=5.5 \mathrm{~Hz}, 2 \mathrm{H}_{7}\right)$, $4.05\left(\mathrm{q}, J=6.3 \mathrm{~Hz}, 1 \mathrm{H}_{13}\right), 3.78\left(\mathrm{~s}, \mathrm{OCH}_{3}\right), 2.15\left(\mathrm{~m}, 2 \mathrm{H}_{10}\right), 2.05(\mathrm{br} \mathrm{s}, \mathrm{OH}), 1.70\left(\mathrm{~m}, 2 \mathrm{H}_{12}\right), 1.55(\mathrm{~m}$, 
New capsaicin and dihydrocapsaicin derivatives as histone deacetylase inhibitors

$\left.1 \mathrm{H}_{16}\right), 1.40\left(\mathrm{~m}, 2 \mathrm{H}_{11}\right), 0.96\left(\mathrm{~s}, 9 \mathrm{H}_{21}\right), 0.92\left(\mathrm{~d}, J=6.0 \mathrm{~Hz}, 3 \mathrm{H}_{17}\right), 0.92\left(\mathrm{~d}, J=6.0 \mathrm{~Hz}, 3 \mathrm{H}_{18}\right), 0.16\left(\mathrm{~s}, 6 \mathrm{H}_{19}\right)$. ${ }^{13} \mathrm{C}$ NMR $\left(\mathrm{CDCl}_{3}\right): \delta 172.70\left(\mathrm{C}_{9}\right), 151.09\left(\mathrm{C}_{3}\right), 144.52\left(\mathrm{C}_{4}\right), 139.19\left(\mathrm{C}_{15}\right), 138.36\left(\mathrm{C}_{14}\right), 129.63\left(\mathrm{C}_{1}\right)$, $120.86\left(\mathrm{C}_{6}\right), 120.18\left(\mathrm{C}_{5}\right), 112.00\left(\mathrm{C}_{2}\right), 72.60\left(\mathrm{C}_{13}\right), 55.50\left(\mathrm{OCH}_{3}\right), 43.54\left(\mathrm{C}_{7}\right), 36.67\left(\mathrm{C}_{10}\right), 31.81\left(\mathrm{C}_{12}\right)$, $29.80\left(\mathrm{C}_{16}\right), 25.69\left(\mathrm{C}_{20}\right), 22.29\left(\mathrm{C}_{18}\right), 22.24\left(\mathrm{C}_{17}\right), 21.56\left(\mathrm{C}_{11}\right), 18.42\left(\mathrm{C}_{21}\right),-4.65\left(\mathrm{C}_{19}\right)$. HRMS $\mathrm{m} / \mathrm{z}$ $458.2704[\mathrm{M}+\mathrm{Na}]^{+}$(calcd. For $\left.\mathrm{C}_{24} \mathrm{H}_{41} \mathrm{NO}_{4} \mathrm{Si}+\mathrm{Na}, 458.2703\right)$.

\subsubsection{Preparation of Allylic Alcohol (6)}

To a solution of compounds $\mathbf{1}$ and $\mathbf{2}(25 \mathrm{mg}, 0.08 \mathrm{mmol})$ in THF $(3 \mathrm{~mL})$ was added $\mathrm{SeO}_{2}(10$ $\mathrm{mg}, 1.2$ equis). Catalytic amount of pyridine $(0.1 \mathrm{~mL})$ was added and the reaction mixture was refluxed for $4 \mathrm{~h}$. Then water $(3 \mathrm{~mL})$ was added and the mixture was extracted with EtOAc $(3 \times 20 \mathrm{~mL})$. The organic layers were combined, washed with water, brine and dried with anhydrous $\mathrm{Na}_{2} \mathrm{SO}_{4}$. The solvent was removed to furnish a crude mixture. Purification of the crude mixture by column chromatography $(1: 1$ $(\mathrm{v} / \mathrm{v})$ EtOAc/hexanes) gave a yellow oil of compound $6(9 \mathrm{mg}, 89 \%)$ and recovered compound 2 (12 $\mathrm{mg}, 96 \%)$.

(E)-5-hydroxy-N-(4-hydroxy-3-methoxybenzyl)-8-methylnon-6-enamide $(6): R_{f}=0.20(1: 1 \quad(v / v)$ EtOAc/hexanes). IR (neat) : 3306, 2927, 1758, 1645, 1515, 1262, $1035 \mathrm{~cm}^{-1} .{ }^{1} \mathrm{H}$ NMR $\left(\mathrm{CDCl}_{3}\right): \delta 6.86$ $\left(\mathrm{d}, J=8.0 \mathrm{~Hz}, 1 \mathrm{H}_{5}\right), 6.80\left(\mathrm{~s}, 1 \mathrm{H}_{2}\right), 6.75\left(\mathrm{~d}, J=8.0 \mathrm{~Hz}, 1 \mathrm{H}_{6}\right), 5.80\left(\mathrm{~s}, 1 \mathrm{H}_{8}\right), 5.70\left(\mathrm{br} \mathrm{s}, \mathrm{OH}_{4}\right), 5.60(\mathrm{~m}$, $\left.1 \mathrm{H}_{14}\right), 5.40\left(\mathrm{~m}, 1 \mathrm{H}_{15}\right), 4.35\left(\mathrm{~d}, J=5.4 \mathrm{~Hz}, 2 \mathrm{H}_{7}\right), 4.05\left(\mathrm{q}, J=7.8 \mathrm{~Hz}, 1 \mathrm{H}_{13}\right), 3.85\left(\mathrm{~s}, \mathrm{OCH}_{3}\right), 2.20(\mathrm{t}, J=$ $\left.7.8 \mathrm{~Hz}, 2 \mathrm{H}_{10}\right), 2.00\left(\mathrm{br} \mathrm{s}, \mathrm{OH}_{13}\right), 1.57\left(\mathrm{~m}, 2 \mathrm{H}_{11}\right), 1.40\left(\mathrm{~m}, 2 \mathrm{H}_{12}\right), 1.29\left(\mathrm{~m}, 1 \mathrm{H}_{16}\right), 0.95(\mathrm{~d}, J=6.6 \mathrm{~Hz}$, $\left.3 \mathrm{H}_{17}\right), 0.95\left(\mathrm{~d}, J=6.6 \mathrm{~Hz}, 3 \mathrm{H}_{18}\right) .{ }^{13} \mathrm{C} \mathrm{NMR}\left(\mathrm{CDCl}_{3}\right): \delta 172.81\left(\mathrm{C}_{9}\right), 146.68\left(\mathrm{C}_{3}\right), 145.12\left(\mathrm{C}_{4}\right), 139.22$ $\left(\mathrm{C}_{15}\right), 138.33\left(\mathrm{C}_{14}\right), 129.57\left(\mathrm{C}_{1}\right), 120.81\left(\mathrm{C}_{6}\right), 114.39\left(\mathrm{C}_{5}\right), 110.73\left(\mathrm{C}_{2}\right), 72.60\left(\mathrm{C}_{13}\right), 55.94\left(\mathrm{OCH}_{3}\right), 43.58$ $\left(\mathrm{C}_{7}\right), 36.62\left(\mathrm{C}_{10}\right), 36.33\left(\mathrm{C}_{12}\right), 29.77\left(\mathrm{C}_{16}\right), 22.22\left(\mathrm{C}_{17}\right), 22.22\left(\mathrm{C}_{18}\right), 21.53\left(\mathrm{C}_{11}\right)$. HRMS m/z 344.1841 $[\mathrm{M}+\mathrm{Na}]^{+}$(calcd. for $\mathrm{C}_{18} \mathrm{H}_{27} \mathrm{NO}_{4}+\mathrm{Na}, 344.1838$ ).

\subsubsection{Preparation of Diacetate (7)}

To a solution of dihydroxy-CAP $6(10.0 \mathrm{mg}, 0.03 \mathrm{mmol})$ in pyridine $(2 \mathrm{~mL})$ was added $\mathrm{Ac}_{2} \mathrm{O}$ ( $0.01 \mathrm{~mL}, 0.098 \mathrm{mmol}, 2.5$ equis). The solution was stirred at room temperature for $29 \mathrm{~h}$. Then water ( 3 $\mathrm{mL})$ was added and the mixture was extracted with EtOAc $(3 \times 20 \mathrm{~mL})$. The combined organic layers were dried and concentrated. Purification of the crude product by column chromatography $(1: 3(\mathrm{v} / \mathrm{v})$ EtOAc/hexanes) gave a yellow oil of compound $7(11 \mathrm{mg}, 86 \%)$.

9-(4-acetoxy-3-methoxybenzylamino)-2-methyl-9-oxononan-4-yl acetate (7): $\mathrm{R}_{f}=0.50(1: 1(\mathrm{v} / \mathrm{v})$ EtOAc/hexanes). IR (neat) : 3335, 2929, 2866, 1634, 1463, 1277, 1156, 1126, $1035 \mathrm{~cm}^{-1}$. ${ }^{1} \mathrm{H}$ NMR $\left(\mathrm{CDCl}_{3}\right): \delta 6.98\left(\mathrm{~d}, J=7.8 \mathrm{~Hz}, 1 \mathrm{H}_{5}\right), 6.90\left(\mathrm{~s}, 1 \mathrm{H}_{2}\right), 6.85\left(\mathrm{~d}, J=7.8 \mathrm{~Hz}, 1 \mathrm{H}_{6}\right), 5.80\left(\mathrm{~s}, 1 \mathrm{H}_{8}\right), 5.65(\mathrm{dd}, J$ $\left.=6.5,15.3 \mathrm{~Hz}, 1 \mathrm{H}_{14}\right), 5.32\left(\mathrm{dd}, J=7.2,15.4 \mathrm{~Hz}, 1 \mathrm{H}_{15}\right), 5.15\left(\mathrm{q}, J=7.8 \mathrm{~Hz}, 1 \mathrm{H}_{13}\right), 4.35(\mathrm{~d}, J=5.6 \mathrm{~Hz}$, $\left.2 \mathrm{H}_{7}\right), 3.75\left(\mathrm{~s}, \mathrm{OCH}_{3}\right), 2.30\left(\mathrm{~s}, 3 \mathrm{H}_{20}\right), 2.18\left(\mathrm{t}, J=7.6 \mathrm{~Hz}, 2 \mathrm{H}_{10}\right), 1.95\left(\mathrm{~s}, 3 \mathrm{H}_{22}\right), 1.60\left(\mathrm{~m}, 2 \mathrm{H}_{12}\right), 1.57(\mathrm{~m}$, $\left.2 \mathrm{H}_{11}\right), 1.20\left(\mathrm{~m}, 1 \mathrm{H}_{16}\right), 0.95\left(\mathrm{~d}, J=6.8 \mathrm{~Hz}, 3 \mathrm{H}_{17}\right), 0.95\left(\mathrm{~d}, J=6.8 \mathrm{~Hz}, 3 \mathrm{H}_{18}\right) .{ }^{13} \mathrm{C} \mathrm{NMR}\left(\mathrm{CDCl}_{3}\right): \delta 172.38$ $\left(\mathrm{C}_{9}\right), 170.47\left(\mathrm{C}_{21}\right), 169.11\left(\mathrm{C}_{19}\right), 151.19\left(\mathrm{C}_{3}\right), 141.48\left(\mathrm{C}_{15}\right), 139.08\left(\mathrm{C}_{4}\right), 137.27\left(\mathrm{C}_{1}\right), 124.87\left(\mathrm{C}_{14}\right)$, $122.84\left(\mathrm{C}_{5}\right), 120.04\left(\mathrm{C}_{6}\right), 112.19\left(\mathrm{C}_{2}\right), 74.38\left(\mathrm{C}_{13}\right), 55.89\left(\mathrm{OCH}_{3}\right), 43.47\left(\mathrm{C}_{7}\right), 36.10\left(\mathrm{C}_{10}\right), 34.06\left(\mathrm{C}_{12}\right)$, $30.65\left(\mathrm{C}_{16}\right), 22.63\left(\mathrm{C}_{11}\right), 22.05\left(\mathrm{C}_{18}\right), 22.05\left(\mathrm{C}_{17}\right), 21.36\left(\mathrm{C}_{22}\right), 20.62\left(\mathrm{C}_{20}\right)$. HRMS m/z 386.1613 [M $\mathrm{Ac}+\mathrm{Na}]^{+}$(calcd. for $\left.\mathrm{C}_{20} \mathrm{H}_{29} \mathrm{NO}_{5}+\mathrm{Na}, 386.1943\right)$.

\subsubsection{Preparation of Chloranil-CAP $(\mathbf{8})$}

To a solution of compounds 1 and $2(15.2 \mathrm{mg}, 0.05 \mathrm{mmol})$ in THF $(4 \mathrm{~mL})$ at $-40{ }^{\circ} \mathrm{C}$ under $\mathrm{N}_{2}$ was added $13.4 \mathrm{mg}(0.05 \mathrm{mmol})$ of $p$-chloranil. After $20 \mathrm{~h}$. at room temperature, the solvent was removed in vacuo and $5 \% \mathrm{NaHCO}_{3}$ was added to the residue which was then extracted with EtOAc (3x20 mL). The combined organic layers were dried and concentrated. Purification of the crude product by column chromatography (1:1 (v/v) EtOAc/hexanes) gave a brown gum of compounds 8 and 9 (16 mg, $95 \%)$. 
(E)-N-(3-methoxy-4-(2,4,5-trichloro-3,6-dioxocyclohexa-1,4-dienyloxy)-benzyl)-8-methylnon-6enamide (8): $\mathrm{R}_{f}=0.75(1: 1(\mathrm{v} / \mathrm{v})$ EtOAc/hexanes). $\mathrm{IR}(\mathrm{KBr}): 3435,3304,2954,2926,2852,1692$, $1644,1572,1505,1195,1175 \mathrm{~cm}^{-1} .{ }^{1} \mathrm{H}$ NMR $\left(\mathrm{CDCl}_{3}\right): \delta 7.05\left(\mathrm{~d}, J=8.6 \mathrm{~Hz}, 1 \mathrm{H}_{5}\right), 6.85\left(\mathrm{~s}, 1 \mathrm{H}_{2}\right), 6.85$ $\left(\mathrm{s}, 1 \mathrm{H}_{6}\right), 5.75\left(\mathrm{br} \mathrm{s}, 1 \mathrm{H}_{8}\right), 5.35\left(\mathrm{~m}, 1 \mathrm{H}_{14}\right), 5.30\left(\mathrm{~m}, 1 \mathrm{H}_{15}\right), 4.40\left(\mathrm{~d}, J=5.4 \mathrm{~Hz}, 2 \mathrm{H}_{7}\right), 3.75\left(\mathrm{~s}, \mathrm{OCH}_{3}\right), 2.20$ (t, $\left.J=7.8 \mathrm{~Hz}, 2 \mathrm{H}_{10}\right), 1.98\left(\mathrm{q}, J=7.2 \mathrm{~Hz}, 2 \mathrm{H}_{13}\right), 1.65$ (quint, $\left.J=7.8 \mathrm{~Hz}, 2 \mathrm{H}_{11}\right), 1.38\left(\mathrm{~m}, 2 \mathrm{H}_{12}\right), 1.29(\mathrm{~m}$, $\left.1 \mathrm{H}_{16}\right), 0.95\left(\mathrm{~d}, J=6.7 \mathrm{~Hz}, 6 \mathrm{H}_{17,18}\right) \cdot{ }^{13} \mathrm{C} \mathrm{NMR}\left(\mathrm{CDCl}_{3}\right): \delta 172.93\left(\mathrm{C}_{9}\right), 171.76\left(\mathrm{C}_{20}\right), 170.64\left(\mathrm{C}_{23}\right), 153.14$ $\left(\mathrm{C}_{19}\right)$, $148.61\left(\mathrm{C}_{3}\right), 144.22\left(\mathrm{C}_{4}\right), 140.42\left(\mathrm{C}_{21}\right), 138.70\left(\mathrm{C}_{22}\right), 138.12\left(\mathrm{C}_{15}\right), 136.31\left(\mathrm{C}_{1}\right), 126.40\left(\mathrm{C}_{14}\right)$, $124.43\left(\mathrm{C}_{24}\right), 120.64\left(\mathrm{C}_{6}\right), 119.14\left(\mathrm{C}_{5}\right), 112.68\left(\mathrm{C}_{2}\right), 56.34\left(\mathrm{OCH}_{3}\right), 43.20\left(\mathrm{C}_{7}\right), 36.64\left(\mathrm{C}_{10}\right), 32.18\left(\mathrm{C}_{16}\right)$, $30.94\left(\mathrm{C}_{13}\right), 29.26\left(\mathrm{C}_{12}\right), 25.21\left(\mathrm{C}_{11}\right), 22.63\left(\mathrm{C}_{17}\right), 22.63\left(\mathrm{C}_{18}\right)$.
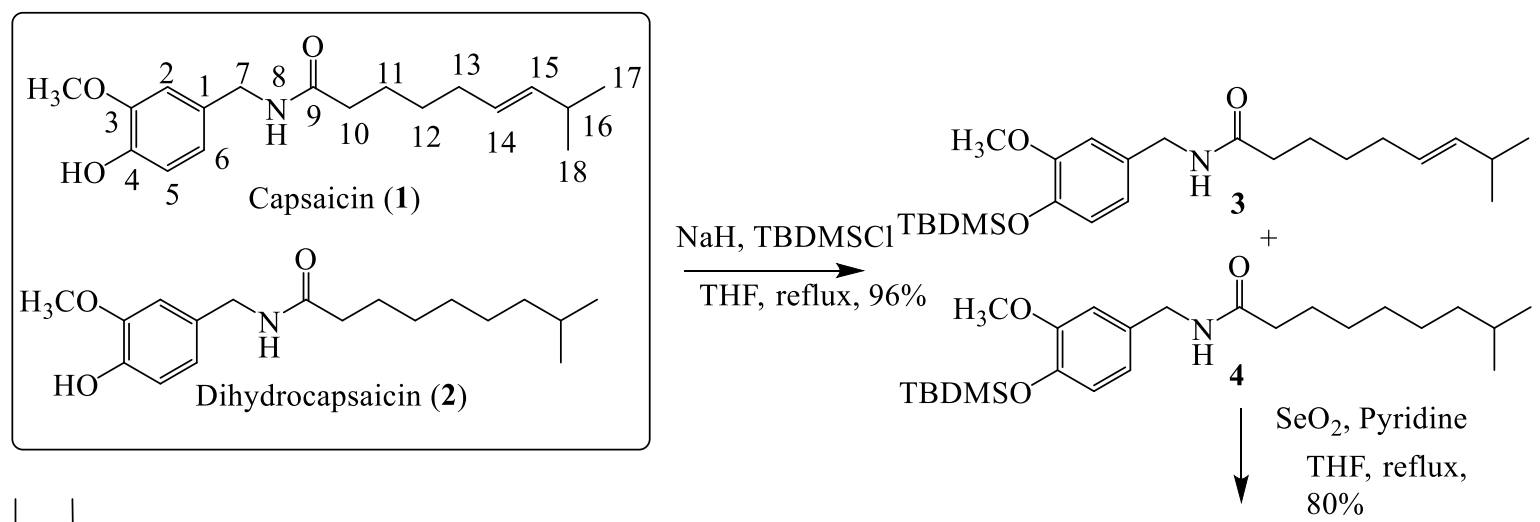

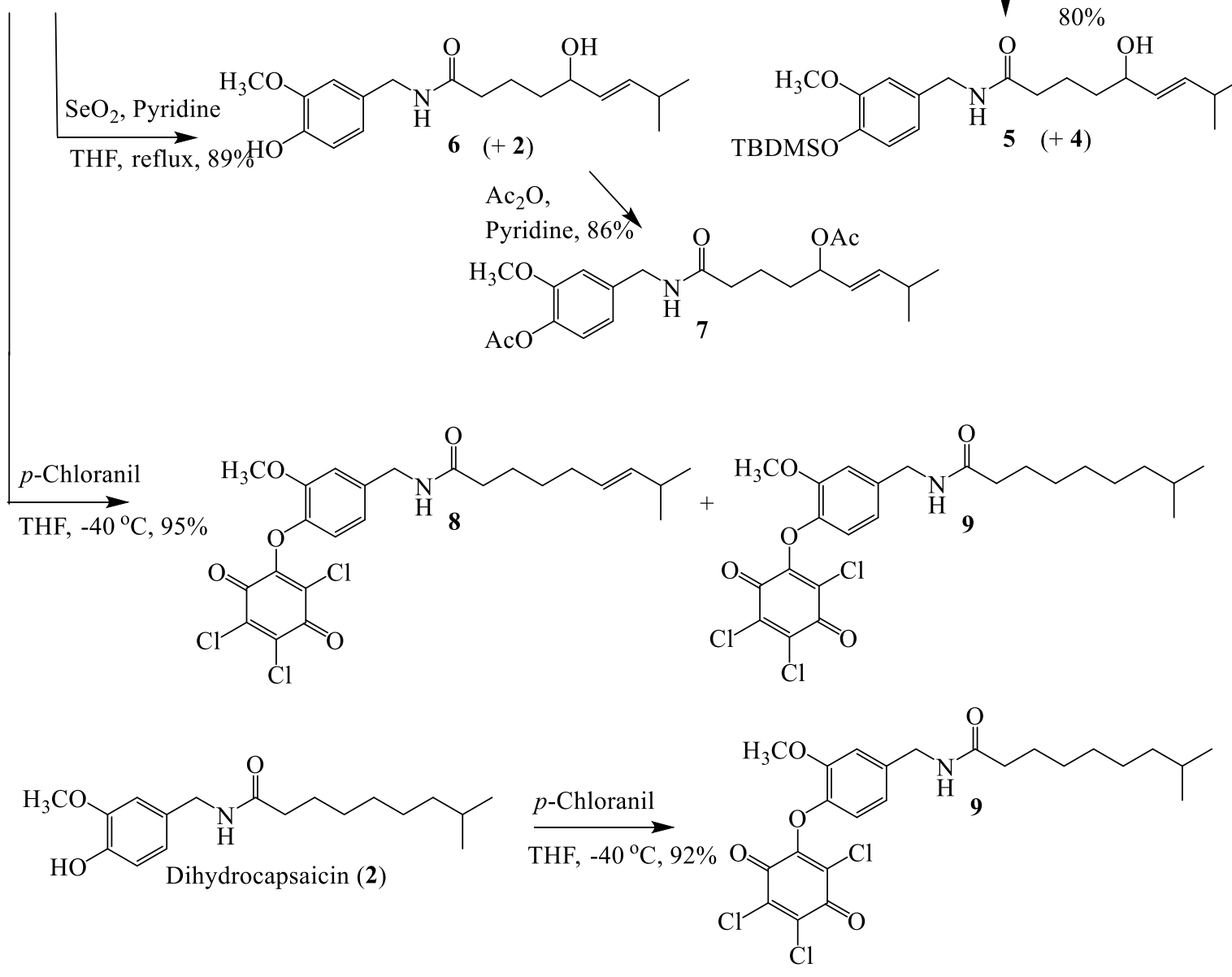

Figure 2. Structural modification of CAP/DHC derivatives 
New capsaicin and dihydrocapsaicin derivatives as histone deacetylase inhibitors

\subsubsection{Preparation of Chloranil-DHC (9)}

To a solution of DHC $2(35.0 \mathrm{mg}, 0.114 \mathrm{mmol})$ in THF $(4 \mathrm{~mL})$ at $-40{ }^{\circ} \mathrm{C}$ under $\mathrm{N}_{2}$ was added $42.0 \mathrm{mg}(0.171 \mathrm{mmol})$ of $p$-chloranil. After $17 \mathrm{~h}$ at room temperature, the solvent was removed in vacuo and $5 \% \mathrm{NaHCO}_{3}$ was added to the residue and the residue was extracted with EtOAc $(3 \times 20 \mathrm{~mL})$. The combined organic layers were dried and concentrated. Purification of the crude product by column chromatography (1:1 (v/v) EtOAc/hexanes) gave a brown gum of compounds 9 (36.3 mg, 92\%).

$N$-(3-methoxy-4-(2,4,5-trichloro-3,6-dioxocyclohexa-1,4-dienyloxy)benzyl)-8-methylnonan-amide (9): $\mathrm{R}_{f}=0.75(1: 1(\mathrm{v} / \mathrm{v})$ EtOAc/hexanes). IR (KBr) : 3299, 2921, 2851, 1692, 1644, 1573, 1535, 1505, 1466, $1431,1366,1321,1259,1195,1147,1123,1059,1035,793,722 \mathrm{~cm}^{-1} .{ }^{1} \mathrm{H}$ NMR $\left(\mathrm{CDCl}_{3}\right): \delta 7.05(\mathrm{~d}, J=$ $\left.7.9 \mathrm{~Hz}, 1 \mathrm{H}_{5}\right), 6.85\left(\mathrm{~s}, 1 \mathrm{H}_{2}\right), 6.84\left(\mathrm{~m}, 1 \mathrm{H}_{6}\right), 6.0\left(\mathrm{br} \mathrm{s}, 1 \mathrm{H}_{8}\right), 4.40\left(\mathrm{~s}, 2 \mathrm{H}_{7}\right), 3.75\left(\mathrm{~s}, \mathrm{OCH}_{3}\right), 2.25(\mathrm{t}, J=7.8$ $\left.\mathrm{Hz}, 2 \mathrm{H}_{10}\right), 1.62$ (quint, $\left.J=6.8 \mathrm{~Hz}, 2 \mathrm{H}_{11}\right), 1.50\left(\mathrm{~m}, 1 \mathrm{H}_{16}\right), 1.30\left(\mathrm{~m}, 2 \mathrm{H}_{12}\right), 1.26\left(\mathrm{~m}, 2 \mathrm{H}_{13}\right), 1.25\left(\mathrm{~m}, 2 \mathrm{H}_{14}\right)$, $1.15\left(\mathrm{~m}, 2 \mathrm{H}_{15}\right), 0.85\left(\mathrm{~d}, J=6.4 \mathrm{~Hz}, 6 \mathrm{H}_{17}, 18\right) .{ }^{13} \mathrm{C} \mathrm{NMR}\left(\mathrm{CDCl}_{3}\right): \delta 173.41\left(\mathrm{C}_{9}\right), 171.75\left(\mathrm{C}_{20}\right), 170.64$ $\left(\mathrm{C}_{23}\right), 153.13\left(\mathrm{C}_{19}\right), 148.61\left(\mathrm{C}_{3}\right), 144.25\left(\mathrm{C}_{4}\right), 140.42\left(\mathrm{C}_{21}\right), 138.69\left(\mathrm{C}_{22}\right), 136.10\left(\mathrm{C}_{1}\right), 124.44\left(\mathrm{C}_{24}\right)$, $120.67\left(\mathrm{C}_{6}\right), 119.14\left(\mathrm{C}_{5}\right), 112.72\left(\mathrm{C}_{2}\right), 56.37\left(\mathrm{OCH}_{3}\right), 43.30\left(\mathrm{C}_{7}\right), 38.92\left(\mathrm{C}_{15}\right), 36.65\left(\mathrm{C}_{10}\right), 29.58\left(\mathrm{C}_{13}\right)$, $29.32\left(\mathrm{C}_{12}\right), 27.91\left(\mathrm{C}_{16}\right), 27.21\left(\mathrm{C}_{14}\right), 25.79\left(\mathrm{C}_{11}\right), 22.60\left(\mathrm{C}_{17}\right), 22.60\left(\mathrm{C}_{18}\right)$. HRMS m/z 538.0966 [M + $\mathrm{Na}]^{+}$(calcd. for $\mathrm{C}_{24} \mathrm{H}_{28} \mathrm{Cl}_{3} \mathrm{NO}_{5}+\mathrm{Na}, 538.0931$ ).

\subsection{Biological Assay}

\subsubsection{Histone Deacetylase Activity Assay}

CAP/DHC and their synthetic derivatives were evaluated for their ability to inhibit a commercially available assay (Fluor de Lys assay system, Biomol, Enzo Life Sciences International, Inc., USA). TSA purchased from Sigma-Aldrich Corporation (USA) was used as the positive control. A $5 \mu \mathrm{L}$ of each sample (in DMSO), $1 \mu \mathrm{L}$ of the Hela nuclear extract and $19 \mu \mathrm{L}$ of a buffer were added into the 96-well plate and incubated at $37{ }^{\circ} \mathrm{C}$ for 5 minutes. Next, a $25 \mu \mathrm{L}$ of a substrate was added and incubated again at $37^{\circ} \mathrm{C}$ for 15 minutes. Deacetylation of the substrate followed with the addition of the developer $(50 \mu \mathrm{L})$ generated a fluorophore. Comparison of inhibitor versus control relative fluorescence signals with excitation at $360 \mathrm{~nm}$ and emission at $460 \mathrm{~nm}$ was done using the spectra Max Gemini XPS microplate spectrofluorometer. All experiments were carried out in triplicate.

\subsubsection{Molecular Docking Studies}

Molecular docking studies were performed for 50 runs using AutoDockTools 1.5.4 (ADT) and AutoDock 4.2 programs with a grid box size of $66 \times 66 \times 66$ points, spacing between grid points as 0.375 angstroms and Lamarckian genetic algorithm search. The crystal structures of HDACs were obtained from the Protein Data Bank [PDB entry code: 4BKX (HDAC1), 3MAX (HDAC2), 4A69 (HDAC3), 2VQW (HDAC4), 6UO2 (HDAC6), 3C0Z (HDAC7) and 1T64 (HDAC8)]. All water and noninteracting ions as well as ligands were removed. Then all missing hydrogen and sidechain atoms were added using the ADT program. Gasteiger charges were calculated for the system. For ligand setup, the molecular modeling program Gauss View 03 program was used to build the ligands. Energy of the ligands was optimized with the AM1 level using Gaussian program.

\section{Results and Discussion}

A mixture of capsaicin (CAP, 1) and dihydrocapsaicin (DHC, 2) was isolated from C. annuum fruits ${ }^{18}$. The structural modification of a CAP/DHC mixture was performed without further purification, as shown in Figure 2. The mixture of $\mathbf{1}$ and $\mathbf{2}$ was protected by TBDMSCl to produce the silyl ether mixture 3 and $\mathbf{4}$ in a good yield. Allylic oxidation of the mixture 3 and $\mathbf{4}$ with $\mathrm{SeO}_{2}$ and pyridine provided allylic alcohol silyl ether $\mathbf{5}$ along with recovery of $\mathbf{4}$. Furthermore, allylic oxidation of the mixture $\mathbf{1}$ and $\mathbf{2}$ also gained allylic alcohol $\mathbf{6}$ in a good yield together with recovery of $\mathbf{2}$. The structure of $\mathbf{6}$ was 
confirmed as the racemic mixture by $\mathrm{CD}$ experiment. The racemic mixture of $\mathbf{6}$ was readily converted into diacetate 7 by using acetic anhydride and pyridine. We attempted to oxidize the phenolic moiety of 1 and 2 with $p$-chloranil. Surprisingly, the mixture of chloranil-CAP 8 and chloranil-DHC 9 was obtained in a high yield. In the same condition, chloranil-DHC $\mathbf{9}$ was achieved by treating $\mathbf{2}$ with $p$ chloranil. The structure of $\mathbf{9}$ was confirmed by mass spectrum.

Table 1. The in vitro HDAC inhibitory activities of the obtained compounds

\begin{tabular}{cc}
\hline Compound & \%HDAC inhibition \\
\hline $\mathbf{1 , 2}$ & Not detected \\
$\mathbf{3 , 4}$ & $83.3 \pm 0.8$ \\
$\mathbf{4}$ & $85.1 \pm 0.9$ \\
$\mathbf{5}$ & $76.2 \pm 0.7$ \\
$\mathbf{6}$ & $85.0 \pm 0.9$ \\
$\mathbf{7}$ & $79.4 \pm 0.8$ \\
$\mathbf{8 , 9}$ & $86.0 \pm 0.6$ \\
$\mathbf{9}$ & $87.0 \pm 0.6$ \\
$\mathbf{T S A}(\mathbf{2 5} \boldsymbol{\mu M})$ & $93.1 \pm 0.8$ \\
\hline
\end{tabular}

CAP 1, DHC 2 and their synthetic derivatives were tested in vitro using a HeLa nuclear extract in a fluoro metric assay at $100 \mu \mathrm{M}$. The inhibitory activities of all compounds against HDAC are presented in Table 1. The mixture of CAP 1 and DHC 2 exhibited no activity. Modification of the phenolic group provided the mixture of silyl ether-CAP $\mathbf{3}$ and silyl ether-DHC $\mathbf{4}$ with high inhibitory activity. The incorporation of hydroxyl group in the alkyl chains was conducted to gain allylic alcohols 5 and $\mathbf{6}$, which were expected to exhibit improved coordinating and chelating properties to the zinc ion. Surprisingly, only allylic alcohol 6 showed high inhibitory activity against HDAC with $85 \%$ inhibition. Allylic alcohol silyl ether $\mathbf{5}$ showed less inhibitory activity against HDAC than the compound $\mathbf{3}$. These data suggested that the silyl group of allylic alcohol silyl ether $\mathbf{5}$ may be too bulky to allow ligandenzyme binding. The HDAC inhibitory activity test of diacetate $\mathbf{7}$ was performed to examine whether the acetate esters in the alkyl side chain and the phenolic improved activity. Unfortunately, diacetate 7 exhibited slightly reduced HDAC inhibition. Chloranil CAP 8 and chloranil DHC 9 showed the most inhibitory activities against HDAC among the tested compounds. The data indicated that high electron intensity of the chloranil group may be chelating to the zinc ion or interacting at the active site cavity.

Table 2. The in silico HDAC inhibitory activities of the obtained compounds

\begin{tabular}{|c|c|c|c|c|c|c|c|c|c|c|c|c|c|c|}
\hline \multirow[t]{3}{*}{ Cp } & \multicolumn{8}{|c|}{ Class I } & \multicolumn{4}{|c|}{ Class IIa } & \multirow{2}{*}{\multicolumn{2}{|c|}{$\begin{array}{l}\text { Class IIb } \\
\text { HDAC6 }\end{array}$}} \\
\hline & \multicolumn{2}{|c|}{ HDAC1 } & \multicolumn{2}{|c|}{ HDAC2 } & \multicolumn{2}{|c|}{ HDAC3 } & \multicolumn{2}{|c|}{ HDAC8 } & \multicolumn{2}{|c|}{ HDAC4 } & \multicolumn{2}{|c|}{ HDAC7 } & & \\
\hline & $\Delta G^{\mathrm{a}}$ & $K \mathbf{i}^{\mathbf{b}}$ & $\Delta G^{\mathrm{a}}$ & $K \mathbf{i}^{\mathbf{b}}$ & $\Delta \boldsymbol{G}^{\mathrm{a}}$ & $K \mathbf{i}^{\mathbf{b}}$ & $\Delta \boldsymbol{G}^{\mathrm{a}}$ & $K \mathbf{i}^{\mathbf{b}}$ & $\Delta G^{\mathbf{a}}$ & $K \mathbf{i}^{\mathbf{b}}$ & $\Delta G^{\mathrm{a}}$ & $K \mathbf{i}^{\mathbf{b}}$ & $\Delta G^{\mathbf{a}}$ & $K \mathbf{i}^{\mathbf{b}}$ \\
\hline 3 & -7.78 & 2.00 & -6.56 & 15.50 & -7.30 & 5.25 & -6.76 & 11.10 & -7.07 & 6.57 & -7.39 & 4.50 & -7.22 & 4.84 \\
\hline 4 & -7.73 & 2.14 & -6.96 & 7.88 & -7.14 & 5.81 & -6.46 & 18.50 & -7.50 & 3.18 & -6.94 & 8.17 & -6.94 & 8.21 \\
\hline 5 & -7.29 & 4.55 & -8.26 & 0.88 & -7.27 & 4.67 & -7.15 & 5.73 & -7.34 & 4.20 & -7.91 & 1.60 & -7.49 & 3.26 \\
\hline 6 & -6.78 & 10.66 & -7.51 & 3.11 & -7.69 & 2.32 & -7.50 & 3.17 & -6.80 & 10.43 & -7.30 & 4.46 & -7.80 & 1.93 \\
\hline 7 & -5.91 & 46.17 & -6.70 & 12.17 & -7.03 & 7.07 & -6.18 & 29.53 & -6.94 & 8.16 & -6.29 & 24.4 & -6.32 & 23.1 \\
\hline 8 & -6.91 & 8.58 & -8.87 & 0.32 & -7.95 & 1.44 & -7.47 & 3.34 & -8.69 & 0.43 & -8.18 & 1.02 & -7.89 & 1.66 \\
\hline 9 & -7.05 & 6.75 & -8.85 & 0.33 & -7.59 & 2.74 & -7.06 & 6.70 & -8.25 & 0.90 & -7.95 & 1.48 & -7.26 & 4.74 \\
\hline
\end{tabular}

${ }^{\mathrm{a}}(\mathrm{kcal} / \mathrm{mol})^{\mathrm{b}}(\mu \mathrm{M})$

Molecular docking studies were conducted with the presentative HDAC Class I (HDAC1, 2, 3, 8), Class IIa (HDAC4, 7) and Class IIb (HDAC6) to gain more details on the binding mode and to obtain 
New capsaicin and dihydrocapsaicin derivatives as histone deacetylase inhibitors

the isoform-selective inhibitors with different HDAC enzymes. The active in vitro compounds were analyzed to allow comparisons of the calculated free energies of binding $(\Delta \mathrm{G})$ and inhibition constants $(\mathrm{Ki})$, which are also demonstrated in Table 2. Silyl ether $\mathbf{3}$ and $\mathbf{4}$ showed moderate inhibition against HDAC2, HDAC8, HDAC4 and HDAC7. Interestingly, silyl ether $\mathbf{3}$ and $\mathbf{4}$ displayed selectivity to HDAC1 with $K \mathrm{i}$ values as 2.00 and $2.14 \mu \mathrm{M}$, respectively. Allylic alcohol 5 was selective towards HDAC2 $(K \mathrm{i}=0.88 \mu \mathrm{M})$. The compound 5-HDAC2 complex presented a zinc chelation via the hydroxyl group and the II-II interaction between the phenyl group of $\mathbf{5}$ and Tyr209 as demonstrated in Figure 3A. The molecular docking results of compound $\mathbf{5}$ also revealed that the methoxy and the silyloxy groups in the aromatic region played a critical role in the surface recognition element. Alcohol $\mathbf{6}$ showed moderate inhibition against HDAC1, HDAC2, HDAC3, HDAC4, HDAC7 and HDAC8. Remarkably, alcohol 6 was selective towards HDAC6 $(\mathrm{Ki}=1.93 \mu \mathrm{M})$. While, diacetate 7 displayed weak to moderate inhibitions against all HDACs. Interestingly, chloranil $\mathbf{8}$ and $\mathbf{9}$ which were the most active HDAC inhibitor in vitro showed a strong inhibitory activity against $\operatorname{HDAC} 2(K \mathrm{i}=0.32,0.33 \mu \mathrm{M})$ and HDAC4 $(K \mathrm{i}=0.43,0.90 \mu \mathrm{M})$, respectively. Figure 3B shows the binding interactions of 8 with the active site cavity of HDAC2.
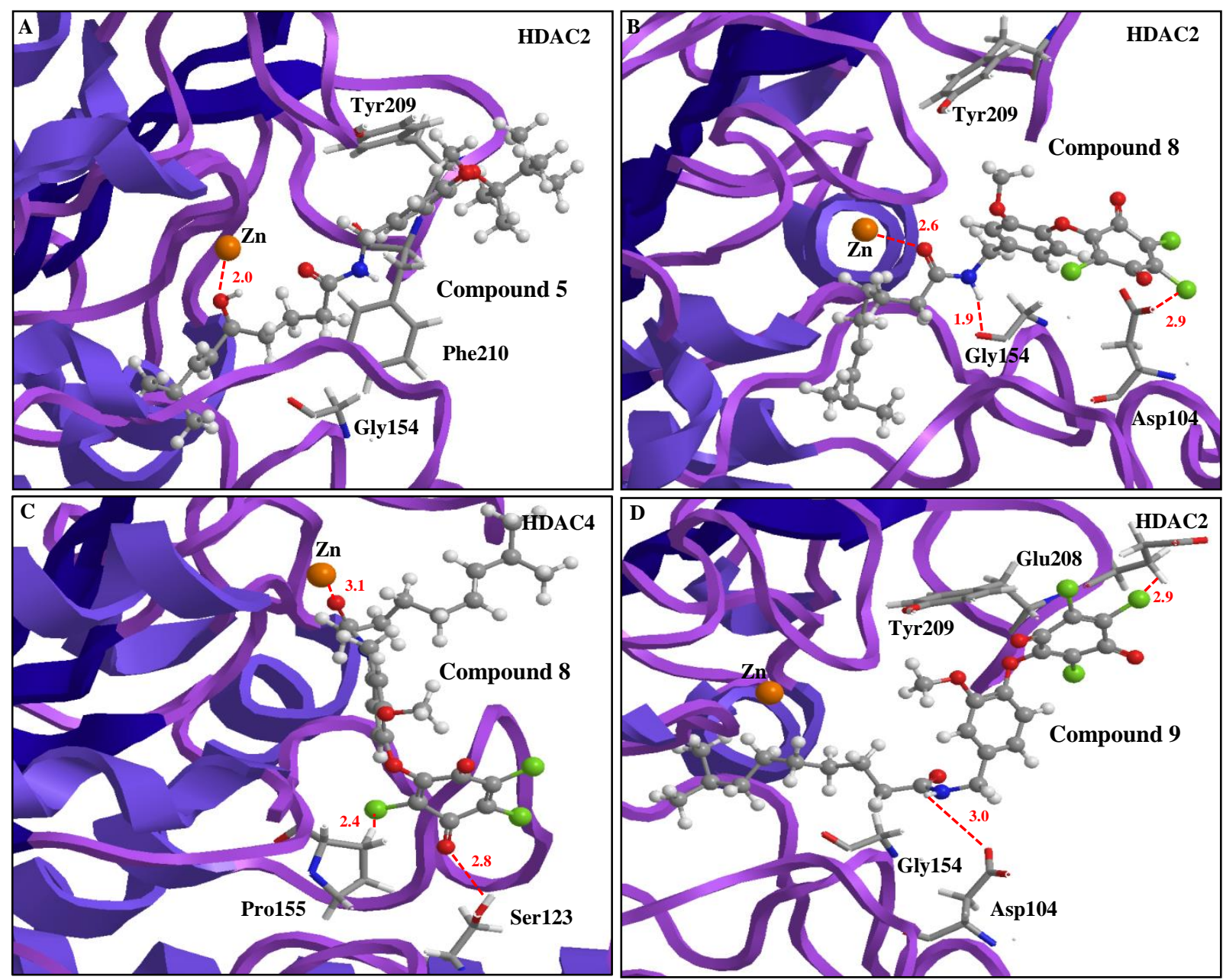

Figure 3. The interaction modes of (A): $\mathbf{5}$ in the active site of HDAC2, (B): $\mathbf{8}$ in the active site of HDAC2, (C): 8 in the active site of HDAC4 and (D): $\mathbf{9}$ in the active site of HDAC2

The compound 8-HDAC2 complex presented a zinc chelation via the carbonyl group (2.6 ̊) and interaction with Asp104. Another interaction was seen between the amide group of $\mathbf{8}$ and Gly154 with a distance of $1.9 \AA$. The compound 8-HDAC4 complex (Figure 3C) displayed a zinc chelation via the carbonyl group $(3.1 \AA)$. Two important interactions considered to be hydrogen bonds occurred: one hydrogen bond occurred between the back bone hydroxyl group of Ser123 and the carbonyl group that 
belongs to $\mathbf{8}(2.8 \AA$ ). Another interaction was seen between the chlorine atom of $\mathbf{8}$ and the hydrogen atom of Pro155. The major interaction between HDAC2 and compound $\mathbf{9}$ were also hydrogen bonds (Figure 3D). One hydrogen bond occurred between the carbonyl group of Asp104 and the amide moiety of 9. Another interaction was seen between the chlorine atom of $\mathbf{9}$ and Glu208.

The HDAC activity and the docking data revealed that CAP and DHC derivatives possessing a chloranil-substitution pattern acted as good HDAC inhibitors with selectivity to HDAC2 and HDAC4. HDAC2 play crucial roles in medulloblastoma, lung, liver and pancreatic cancers ${ }^{19-21}$. HDAC4 play a significant role in colon, glioblastoma, ovarian and gastric cancers ${ }^{22-24}$. Therefore, these compounds could be energetic leads for safe and selective anticancer agents with HDAC inhibitory activity.

\section{Conclusion}

A series of new capsaicin and dihydrocapsaicin derivatives were designed and synthesized with the aim to improve the HDAC inhibitory activities by converting the surface-recognition domain and installing the hydroxyl group at the metal-binding affinity. Seven derivatives exhibited inhibitory activity against HDAC in the micromolar concentration range. A conventional modification of the phenolic group into a silyl ether, acetyl ester, and chloranil resulted in an improved inhibitory activity compared to that of the lead compounds. Incorporation of the hydroxy group into the alkyl side chain provided alternative coordination of the zinc ion and resulted in the increased activity. The molecular docking studies provided valuable information with selective HDAC isoforms. These obtained data show that minor structural changes in capsaicin and dihydrocapsaicin can significantly improve both HDAC inhibitory and anticancer activities. These novel non-hydroxamic acid HDAC inhibitors should be further studied and developed as anticancer drugs.

\section{Acknowledgements}

This work was supported by Rajamangala University of Technology Isan (RMUTI).We also would like to thank Mr. Kittisak Poopasith for the excellent NMR data.

\section{Supporting Information}

Supporting information accompanies this paper on http://www.acgpubs.org/journal/organic$\underline{\text { communications }}$

\section{ORCID}

Pakit Kumboonma: 0000-0002-4726-5191

Somprasong Saenglee: 0000-0001-6950-0052

Thanaset Senawong: 0000-0001-8788-5291

Chanokbhorn Phaosiri: 0000-0002-0108-0204

\section{References}

[1] Paris, M.; Porcelloni, M.; Binaschi, M.; Fattori, D. Histone deacetylase inhibitors: from bench to clinic. J. Med. Chem. 2008, 51, 1505-1529.

[2] Noh, H.; Oh, E. Y.; Seo, J. Y.; Yu, M. R.; Kim, Y. O.; Ha, H.; Lee, H. B. Histone deacetylase-2 is a key regulator of diabetes and transforming growth factor- $\beta 1$-induced renal injury. Am. J. Physiol. Renal. Physiol. 2009, 207, 729-739.

[3] Bertrand, P. Inside HDAC with HDAC inhibitors. Eur. J. Med. Chem. 2010, 45, 2095-2116.

[4] Manal, M.; Chandrasekar, M. J.; Gomathi Priya, J.; Nanjan, M. J. Inhibitors of histone deacetylase as antitumor agents: A critical review. Bioorg. Chem. 2016, 67, 18-42.

[5] Micelli, C.; Rastelli, G. Histone deacetylases: structural determinants of inhibitor selectivity. Drug Discov. Today 2015, 20, 718-735. 
New capsaicin and dihydrocapsaicin derivatives as histone deacetylase inhibitors

[6] Bartling, B.; Hofmann, H. S.; Boettger, T., Hansen, G.; Burdach, S.; Silber, R. E.; Simm, A. Comparative application of antibody and gene array for expression profiling in human squamous cell lung carcinoma. Lung Cancer 2005, 49, 145-154.

[7] Khabele, D.; Son, D. S.; Parl, A. K.; Goldberg, G. L.; Augenlicht, L. H.; Mariadason, J. M.; Rice, V. M. Drug-induced inactivation or gene silencing of class I histone deacetylase suppresses ovarian cancer cell growth: Implication for therapy. Cancer Biol. Ther. 2007, 6, 795-801.

[8] Oehme, I.; Deubzer, H. E.; Wegener, D.; Pickert, D.; Linke, J. P.; Hero, B.; Kopp-Schneider, A.; Westermann, F.; Ulrich, S. M.; von Deimling, A.; Fisher, M.; Witt, O. Histone deacetylase 8 in neuroblastoma tumorigenesis. Clin. Cancer Res. 2009, 15, 91-99.

[9] Rettig, I.; Koeneke, E.; Trippel, F.; Mueller, W. C.; Burhenne, J.; Kopp-Schneider, A.; Fabian, J.; Schober, A.; Fernekorn, U.; von Deimling, A.; Deubzer, H. E.; Milde, T.; Witt, O.; Oehme, I. Selective inhibition of HDAC8 decreases neuroblastoma growth in vitro and in vivo and enhances retinoic acidmediated differentiation. Cell Death. Dis. 2015, 6, e1657.

[10] Guan, J. S.; Haggarty, S. J.; Giacometti, E.; Dannenberg, J. H.; Joseph, N.; Gao, J.; Nieland, T. J.; Zhou, Y.; Wang, X.; Mazitschek, R.; Bradner, J. E.; De Pinho, R. A.; Jaenisch, R.; Tsai, L. H. HDAC2 negatively regulates memory formation and synaptic plasticity. Nature 2009, 459, 55-60.

[11] Song, J.; Noh, J. H.; Lee, J. H.; Eun, J. W.; Ahn, Y. M.; Kim, S. Y.; Lee, S. H.; Park, S. W.; Yoo, N. J.; Lee, J. Y.; Nam, S.W. Increased expression of histone deacetylase 2 is found in human gastric cancer. APMIS 2005, 113, 264-268.

[12] Damaskos, C.; Karatzas, T.; Nikolidakis, L.; Kostakis, I. D.; Garmpi, A.; Karamaroudis, S.; Boutsikos, G.; Damaskou, Z.; Kostakis, A.; Kouraklis, G. Histone deacetylase (HDAC) inhibitors: Current evidence for therapeutic activities in pancreatic cancer. Anticancer Res. 2015, 35, 3129-3135.

[13] Lai, F.; Jin, L.; Gallagher, S.; Mijatov, B.; Zhang, X. D.; Hersey, P. Histone deacetylases (HDACs) as mediators of resistance to apoptosis in melanoma and as targets for combination therapy with selective BRAF inhibitors. Adv. Pharmacol. 2012, 65, 27-43.

[14] Wilson, A. J.; Byun, D. S.; Nasser, S.; Murray, L. B.; Ayyanar, K.; Arango, D.; Figueroa, M.; Meinick, A.; Kao, G. D.; Augenlicht, L. H.; Mariadason, J. M. HDAC4 promotes growth of colon cancer cells via repression of p21. Mol. Biol. Cell. 2008, 19, 4062-4075.

[15] Shen, C. C.; Hu, L. Y.; Yang, A. C.; Chiang, Y. Y.; Hung, J. H.; Tsai, S. J. Risk of uterine, ovarian and breast cancer following pelvic inflammatory disease: a nationwide population-based retrospective cohort study. BMC Cancer 2016, 16, 839 .

[16] Kang, Y. K.; Muro, K.; Ryu, M. H.; Yasui, H.; Nishina, T.; Ryoo, B. Y.; Kamiya, Y.; Akinaga, S.; Boku, N. A phase II trial of a selective c-Met inhibitor tivantinib (ARQ 197) monotherapy as a second- or thirdline therapy in the patients with metastatic gastric cancer. Invest. New Drugs 2014, 32, 355-361.

[17] Chang, S.; Young, B. D.; Li, S.; Qi, X.; Richardson, J.; Olsen, E. N. Histone deacetylase 7 maintains vascular integrity by repressing matrix metalloproteinase 10. Cell 2006, 126, 321-334.

[18] Kumboonma, P.; Phaosiri, C.; Misuna, S.; Senawong, T.; Yenjai, C. Isolation and structural modification of Capsaicin and Dihydrocapsaicin from Capsicum anпuum sp. PACCON2009. 2009, 397-400.

[19] Ecker, J.; Oehme, I.; Mazitschek, R.; Korshunov, A.; Kool, M.; Hielscher, T.; Kiss, J.; Selt, F.; Konrad, C.; Lodrini, M.; Deubzer, H. E.; von Deimling, A.; Kulozik, A. E.; Pfister, S. M.; Witt, O.; Milde, T. Targeting class I histone deacetylase 2 in MYC amplified group 3 medulloblastoma. Acta. Neuropathol. Commun. 2015, 3, 22.

[20] Weichert, W.; Roske, A.; Gekeler, V.; Beckers, T.; Ebert, M. P.; Pross, M.; Dietel, M.; Denkert, C.; Rocken, C. Association of patterns of class I histone deacetylase expression with patient prognosis in gastric cancer: A retrospective analysis. Lancet. Oncol. 2008, 9, 139-148.

[21] Buurman, R.; Gurlevik, E.; Schaffer, V.; Eilers, M.; Sandbothe, M.; Kreipe, H.; Wilkens, L.; Schlegelberger, B.; Kuhnel, F.; Skawran, B. Histone deacetylases activate hepatocyte growth factor signaling by repressing microRNA-449 in hepatocellular carcinoma cells. Gastroenterology 2012, 143, 811-820.

[22] Mottet, D.; Pirotte, S.; Lamour, V.; Hagedorn, M.; Javerzat, S.; Bikfalvi, A.; Bellahcène, A.; Verdin, E.; Castronovo, V. HDAC4 represses p21WAF1/Cip1 expression in human cancer cells through a Sp1dependent, p53-independent mechanism. Oncogene 2009, 28, 243-256. 
Kumboonma et al., Org. Commun. (2021) 14:2 133-143

[23] Shen, Y. F.; Wei, A. M.; Kou, Q.; Zhu, Q. Y.; Zhang, L. Histone deacetylase 4 increases progressive epithelial ovarian cancer cells via repression of $\mathrm{p} 21$ on fibrillar collagen matrices. Oncol. Rep. 2016, 35, 948-954.

[24] Kang, Z. H.; Wang, C. Y.; Zhang, W. L.; Zhang, J. T.; Yuan, C. H.; Zhao, P. W.; Lin, Y. Y.; Hong, S.; Li, C. Y.; Wang, L. Histone deacetylase HDAC4 promotes gastric cancer SGC-7901 cells progression via p21 repression. PLoS One 2014, 9, e98894.

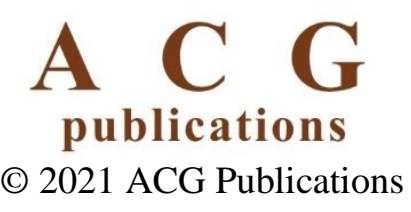

